\title{
Cartas de referência do crescimento somático de crianças dos seis aos 10 anos de idade do Concelho da Maia, Portugal
}

CDD. 20.ed. 613.7

796.07
Mafalda Sofia RORIZ*

André SEABRA*

Rui GARGANTA*

José MAIA*
*Faculdade de Desporto, Universidade do Porto - Portugal.

\section{Resumo}

Os propósitos do estudo foram construir cartas percentílicas para a altura, peso, índice de massa corporal (IMC) e perímetro da cintura (PC) das crianças do Concelho da Maia, Portugal; e contrastar os resultados encontrados no Concelho da Maia com os de outros estudos realizados em Portugal e com os de referência do Center for Disease Control and Prevention (CDC). A amostra é constituída por 3749 crianças (1942 meninos e 1807 meninas) dos seis aos 10 anos de idade. As cartas foram construídas separadamente para cada sexo utilizando o método LMS. A altura, peso, IMC e PC das crianças Maiatas aumentam de modo linear e não linear com a idade. Os Maiatos mostraram valores médios de altura, peso e IMC consistentemente superiores às do CDC. As trajetórias modais do PC dos Maiatos são superiores às de outros estudos internacionais. Observam-se diferenças nas variáveis somáticas em locais e regiões distintas de Portugal.

UnIteRMos: Cartas percentílicas; Altura; Peso; IMC; Perímetro da cintura.

\section{Introdução}

O crescimento físico do ser humano é um processo altamente complexo face à sua plasticidade adaptativa, expressa na variabilidade intra e interindividual, reflexo da ação e interação dos genes e das condiçôes ambientais, sociais, econômicas e culturais (BIELICKI, 1986; UlIJASZEK, 2006). Contudo, ninguém, em lado algum do mundo civilizado, dispensa informação acerca do modo como crianças e jovens crescem, dado que esta informação é um indicador excelente de aspetos da justeza de políticas públicas dirigidas ao desenvolvimento saudável e harmonioso (De Onis, Garza, Victora, Bhan \& Norum, 2004; EVEleth \& TANNER, 1990).

As cartas de referência, ilustração adequada das curvas percentílicas (também expressas em valores z) de crescimento de crianças e jovens, estão entre os instrumentos mais preciosos para a monitorização de aspectos do seu estado nutricional, cuja aplicação é inquestionável nas áreas da Saúde Pública, Pediatria, Nutrição e Ciências do Desporto (DE OnIS, Onyango, Borghi, Siyam, Nishida \& Siekmann, 2007; SOUSA \& MAIA, 2005). De salientar que muitas das intervençóes destinadas a melhorar a saúde das crianças estão dependentes, também, da informação providenciada pelas curvas de crescimento da altura, peso, índice de massa corporal (IMC) e perímetro da cintura (PC) que representam, indiscutivelmente, ferramentas eficazes não só para descrever aspectos da trajetória modal e da variabilidade inter-individual, mas também para identificar eventuais incrementos excessivos, diagnosticar desnutrição, ou ainda atrasos no crescimento linear (Silva, Maia, Claessens, BeUnen \& Pan, 2011).

O incremento do excesso de peso e da obesidade na população infantil é um problema de saúde pública à escala mundial (CENTER FOR DisEASE CONTROL AND PREVENTION - CDC, 2006), de tal forma "assustadora" que a Internacional Obesity Task Force (IOTF) alertou os serviços de vigilância epidemiológica de diversos países, incluindo Portugal, face às prevalências de sobrepeso e obesidade excederem os $30 \%$ em crianças dos sete aos 11 anos (DE OnIS, BLOSSNER, \& BORGHI, 2010; IOTF, 2005; Pereira, Seabra, Silva, KatzmarzyK, BEUNEN \& MAIA, 2010). Muitas crianças e adolescentes 
com excesso de peso e obesidade vão permanecer adultos com excesso de peso e obesidade (JuHOLA, Magnussen, ViIKARI, KäHÖnen, Hutri-KäHÖnen, Jula, Lehtimäki, Akerblom, Pietikäinen, Laitinen, Jokinen, Taittonen, Raitakari \& Juonala, 2011), antecipando desde logo algumas das complicações outrora só observáveis na idade adulta, como a diabetes tipo II, pelo que a utilização das curvas de IMC e do PC têm vindo a assumir uma relevância redobrada na identificação e prevenção de tais efeitos. De realçar que o PC, em relação ao IMC, tem sido frequentemente referido como um indicador relativamente consistente de risco cardiometabólico (JANSSEN, KaTZMARZYK \& Ross, 2004; Lofren, Herron, Zern, West, Patalay, Shachter, Koo \& Fernandez, 2004). Não é pois de estranhar que seja considerado um indicador chave, em crianças e em adultos, da referida síndrome metabólica (Alberti, Zimmet \& Shaw, 2006; Zimmet, Alberti, Kaufman, Tajima, Silink, Arslanian, Wong, Bennett, SHaW \& CAPRIO, 2007).

Apesar de vários países industrializados possuírem informação detalhada acerca do modo como crescem as crianças e os jovens (ver por exemplo a informação do Centro de Prevenção e Controle de Doenças dos EUA: www.cdc.gov ou; da Fundação Inglesa para o Crescimento da Criança: www.childgrowthfoundation.org), a verdade é que a coleta destes dados é uma tarefa logisticamente complexa, morosa e dispendiosa de implementar, que exige elevados recursos humanos, econômicos e materiais.

Portugal é um país com 10,5 milhōes de habitantes (Instituto Nacional de Estatística, IP (INE), 2011) e atualmente ainda não possui cartas de referência nacional para o crescimento somático, e muito especificamente para as idades compreendidas entre os seis e os 10 anos, cuja relevância no domínio da saúde escolar é por demais evidente. Parece haver algum esquecimento por parte dos intervenientes e responsáveis de saúde pediátrica relativamente ao $1^{\circ}$ Ciclo do Ensino Básio $\left(1^{\circ} \mathrm{CEB}\right)$ tão rico de conteúdo informacional. Não obstante este fato, existe já alguma informação disponível e relevante proveniente de diferentes locais e regiōes, a destacar: Amarante (SOUSA \& MaIA, 2005), Viana do Castelo (Rodrigues, Bezerra \& Saraiva, 2007) e Regiōes Autônomas dos Arquipélagos dos Açores (MAIA \& Lopes, 2002, 2007) e da Madeira (Freitas, Maia, Beunen, Claessens, Thomis, Marques, Rodrigues, Silva \& CRESPo, 2002). No espaço desta informação não é clara uma tendência inequívoca dos valores. As regiōes do país não são todas iguais, e na mesma região também se verifica alguma heterogeneidade. Autores como Goldstein e Tanner (1980) e Johnston, Bogin, MacVean e Newman (1984) defenderam com vigor, há mais de 30 anos, a construção e utilização de referências locais para uso clínico, principalmente na avaliação do estado nutricional, por reproduzirem com maior rigor a realidade local.

Neste sentido, o presente estudo vai incidir sobre o Concelho da Maia. Considerando os dados do Instituto Nacional de Estatística (INE, 2009), a Maia é uma cidade de Portugal Continental situada na Região do Norte fazendo parte da Área Metropolitana do Porto. É sede de um município com $83,14 \mathrm{~km}^{2}$ de área e 135.049 habitantes. A presente pesquisa é assim percorrida pelos seguintes objetivos: 1) construir um banco de dados e respectivas cartas de referência percentílica para a altura, peso, IMC e PC das crianças, com idades compreendidas entre os seis e os 10 anos, do Concelho da Maia, Portugal; 2) contrastar os resultados encontrados no Concelho da Maia com os de outros estudos realizados em Portugal e com os de referência do CDC.

\section{Método}

A amostra deste estudo é proveniente do "Projeto Maia Saudável” que conjuga, simultaneamente, um delineamento longitudinal e outro de natureza transversal sobre o crescimento somático, composição corporal, somatótipo, atividade física, desempenho motor e síndrome metabólica de crianças do Concelho da Maia, região situada no norte de Portugal. Duas sub-amostras distintas foram consideradas neste estudo: a primeira decorre da $1^{a}$ fase do delineamento longitudinal cuja informação foi colhida em dois anos letivos consecutivos: 2007/2008 ( $\mathrm{n}=$
949), 2008/2009 ( $\mathrm{n}=1401)$ em crianças dos seis aos 10 anos de idade; a segunda é proveniente do estudo transversal, realizado no ano letivo de 2010/2011, e contém crianças dos oito aos 10 anos de idade $(\mathrm{n}=$ 1399). A amostra total compreende 3749 crianças, 1942 meninos e 1807 meninas (TABELA 1) de um universo de 5039 crianças (a amostra representa $74,4 \%$ da população escolar deste ciclo de ensino). A idade decimal foi obtida a partir da diferença entre a data da coleta da informação e a data de nascimento. Os grupos etários foram constituídos do seguinte 
modo: crianças com idades compreendidas entre 6,00 e 6,99 anos pertencem ao grupo dos seis anos de idade (6+) e assim sucessivamente até $10+$.

TABELA 1 - Distribuição da amostra por sexo e idade.

\begin{tabular}{lccc}
\hline Idades & Meninas & Meninos & Total \\
\hline $6+$ & 214 & 222 & 436 \\
$7+$ & 312 & 306 & 618 \\
$8+$ & 523 & 595 & 1118 \\
$9+$ & 618 & 642 & 1260 \\
$10+$ & 140 & 177 & 317 \\
\hline Total & 1807 & 1942 & 3749 \\
\hline
\end{tabular}

De referir que a seleção da amostra deste estudo obedeceu a critérios de localização geográfica, onde se procurou cobrir todas as freguesias do Concelho da Maia ( $n=17)$ e agrupamentos escolares $(n=7)$, representando de modo aleatório e proporcional idades e sexos, bem como abranger todas as escolas que fizeram parte do estudo de 1997 (Pereira, 2000). Participaram, pois, 22 escolas do $1^{\circ}$ Ciclo do Ensino Básico (CEB) (de um total de 41 escolas) do Concelho da Maia. Foi solicitada autorização prévia à Direção Regional de Educação do Norte (DREN), assim como a todos agrupamentos escolares, já que as avaliações decorreram durante o horário escolar. Os encarregados de educação e as crianças envolvidas na pesquisa assinaram um consentimento informado e o projeto foi aprovado pela Faculdade de Desporto da Universidade do Porto.

\section{Medidas antropométricas}

A estatura foi medida com um antropômetro portátil da marca Siber Hegner ${ }^{\circledR}$, com precisão de 0,1 centímetro $(\mathrm{cm})$, de acordo com as referências de Lohman, Roche e Martorell (1988). O peso foi medido com uma balança de bioimpedância marca Tanita BC-418 $\mathrm{MA}^{\circledR}$ e o resultado expresso em quilogramas $(\mathrm{kg})$ com aproximação ao hectograma. O IMC foi calculado por meio da razão entre o peso e a estatura $\left[\mathrm{Peso}(\mathrm{kg}) / \operatorname{Altura}^{2}(\mathrm{~m})\right]$, obtendo um valor final expresso em $\mathrm{kg} / \mathrm{m}^{2}$. O PC foi medido com uma fita métrica marca Holtain ${ }^{\circledR}$ e registado em centímetros com base no protocolo proposto pelo International Working Group on Kinanthropometry descrito por Ross e MARfELL-Jones (1983).

\section{Controle de qualidade da informação}

A equipe de avaliadores foi sujeita a um treino específico, procurando obter o máximo de rigor na localização dos pontos anatômicos de referência, conhecimento dos procedimentos e técnicas de medida, sobretudo no cumprimento estrito dos protocolos de medição. Os avaliadores foram distribuídos por estaçôes fixas (sempre os mesmos) para cada teste/medição. Os instrumentos foram devidamente verificados e calibrados antes do início das avaliaçôes. Durante a coleta dos dados foram efetuadas retestagens aleatórias em 189 crianças e calculados o erro técnico de medida (ETM) e o coeficiente de variação $(\mathrm{CV})$ das seguintes medidas: altura $(\mathrm{ETM}=$ $0,14 \mathrm{~cm}, \mathrm{CV}=0,11 \%)$; peso $(\mathrm{ETM}=0,13 \mathrm{~kg}, \mathrm{CV}=$ 0,39\%); e PC (ETM = 1,19 cm, CV=1,92\%).

\section{Procedimentos estatísticos}

As cartas percentílicas para a altura, peso, IMC e PC foram construídas separadamente para cada sexo utilizando o modelo matemático LMS (CoLE, Freeman \& Preece, 1998; Cole \& Green, 1992) implementado no "software" LMSchartmaker Pro versão 2.45 (PAN \& Cole, 2011). Todas as observações cujos valores $Z$ fossem inferiores a -4 ou superiores $\mathrm{a}+4$ foram eliminadas (PAN \& Cole, 2004). O método LMS assume que para dados independentes com valores positivos, a transformação Box-Cox específica que cada valor discreto de idade pode ser um meio para normalizar a distribuição dos valores de cada uma das variáveis; os valores $\mathrm{L}, \mathrm{M}$ e S são "Cubic Splines” em cada intervalo etário. São produzidas três curvas suavizadas e específicas de cada idade, denominadas de curva L (transformação "BoxCox"), curva M (mediana) e curva S (coeficiente de variação), com base na seguinte equação,

$$
C_{100 \alpha}(t)=M(t)\left[1+L(t) S(t) Z_{\alpha}\right]^{1 / L(t)}
$$

em que $Z_{\alpha}$ é o desvio normal equivalente para a amostra total, $\alpha$ e $C_{100 \alpha}(t)$ o percentil correspondente. A complexidade do alinhamento de cada curva é medida através dos graus de liberdade equivalentes para $L(t), M(t)$ e $S(t)$. O número apropriado dos graus de liberdade foi selecionado de acordo com as sugestôes de PAN e COLE (2004), baseadas no valor da Deviance (COLE \& GREEN, 1992) e nas representaçōes gráficas do "Q-test" e "Worm-plots" (RoYsTON \& Wright, 2000; VAN BuUREN \& Fredriks, 2001). O "Q-test" mostra a qualidade do ajustamento/modelo, sendo este considerado adequado se as curvas L, $\mathrm{M}$ e $\mathrm{S}$ se situarem entre $-2 \mathrm{e}+2$. O "Worm-plot" é um instrumento de diagnóstico do resultado da modelação das curvas de referência do crescimento. 
O processo de construção das curvas de crescimento envolveu, basicamente, cinco etapas: 1) entrada de dados e controle da sua qualidade; 2) ajustamento de modelos; 3) visualização gráfica a partir das curvas produzidas (L, M e S, bem como os percentis); 4) verificação da qualidade do modelo (diminuição da "Deviance", valores do "Q-test" e "Worm-plot"); 5) com os últimos quatro estágios repetidos tantas vezes quantas necessárias até se atingir um modelo parcimonioso. Para a descrição do melhor modelo LMS, ou seja, dos graus de liberdade mais apropriados equivalentes para as curvas $\mathrm{L}, \mathrm{M}$ e $\mathrm{S}$ usamos a

\section{Resultados}

Os melhores modelos LMS das cartas percentílicas da altura, peso, IMC e PC foram, respectivamente, para as meninas 3053o, 3053o, 3042o e 2053o; e para os meninos 3054o, 3053o, 2053o e 3053o.

A TABELA 2 apresenta os valores percentílicos $\left(\mathrm{P}_{3}\right.$, $\mathrm{P}_{10}, \mathrm{P}_{25}, \mathrm{P}_{50}, \mathrm{P}_{75}, \mathrm{P}_{90}$ e $\left.\mathrm{P}_{97}\right)$ da altura, peso, IMC e PC das crianças dos seis aos 10 anos de idade de ambos os sexos. As respectivas cartas de referência estão na FIGURA 1. O ANEXO I disponibiliza os valores das curvas L, M e S e respectivos erros-padrão.

Como seria de esperar, observam-se incrementos na altura, peso, IMC e no PC ao longo de todas as idades em ambos os sexos. No valor mediano $\left(\mathrm{P}_{50}\right)$ da altura, as meninas são ligeiramente mais baixas que os meninos, apesar da diferença diminuir com o avançar da idade, de tal modo que a situação se inverte aos 10 anos (sugerindo o início do "takeoff" associado ao salto pubertário). As meninas apresentam incrementos na altura dos seis aos 10 anos de $23,32 \mathrm{~cm}$ e os meninos de $21,15 \mathrm{~cm}$. As meninas são menos pesadas que os meninos, com exceção aos 10 anos em que no $\mathrm{P}_{50}$ pesam $+1 \mathrm{~kg}$. No IMC, do notação abreviada "LMMSX"; onde "L" significa EL, "MM" para EM, S para ES e onde "X" é a opção de transformação dos valores da idade sendo "o" se idade original, " $\mathrm{r}$ " se re-escalada $\mathrm{e}$ " $\mathrm{t}$ " se transformada (VAN BuUren \& Fredriks, 2001). A título de exemplo o modelo LMS 2065o significa: 2 graus de liberdade para a curva L, 6 para a curva $M, 5$ para a curva $S$ e a idade foi mantida nos seus valores originais.

$\mathrm{Na}$ comparação de resultados entre as crianças da Maia e os valores do CDC recorreu-se ao teste da média assumindo como referência (valor populacional) os valores do CDC.

percentil 50 ao 97 , as meninas apresentam sempre valores superiores, apesar da média $\left(\mathrm{P}_{50}\right)$ ser muito próxima, com uma variação entre $\pm 0,06 \mathrm{~kg} / \mathrm{m}^{2} \mathrm{e} \pm$ $0,17 \mathrm{~kg} / \mathrm{m}^{2}$. O PC é o que apresenta diferenças mais expressivas entre os sexos, sendo que os meninos têm sempre valores superiores.

A TABELA 3 ilustra o comportamento da média da altura, peso e IMC entre a amostra da Maia e os valores de referência (P50) do CDC. As crianças da Maia são mais altas, dos que as do CDC com exceção aos 10 anos. $\mathrm{O}$ valor médio da altura aos seis e 10 anos de idade é respetivamente nas meninas Maiatas de $119,9 \mathrm{~cm}$ e $140,8 \mathrm{~cm}$ e no CDC de $117,5 \mathrm{~cm}$ e $141,2 \mathrm{~cm}$; e nos meninos maiatos é de $121,3 \mathrm{~cm}$ e $141,0 \mathrm{~cm}$ e no CDC de $118,6 \mathrm{~cm}$ e $141,2 \mathrm{~cm}$. No que se refere ao peso e ao IMC, as crianças Maiatas apresentam valores médios superiores em todas as idades e em ambos os sexos. No peso, nos dois sexos, a diferença entre as médias vai desde os $+3,0 \mathrm{~kg}$ aos seis anos, até aos $+4,4 \mathrm{~kg}$ aos 10 anos. No IMC, a diferença das médias, aos 10 anos de idade é igual em ambos os sexos, isto é $2,1 \mathrm{~kg} / \mathrm{m}^{2}$. 
TABELA 2 -Valores numéricos dos percentis $\left(\mathrm{P}_{3}, \mathrm{P}_{10}, \mathrm{P}_{25}, \mathrm{P}_{50}, \mathrm{P}_{75}, \mathrm{P}_{90}, \mathrm{P}_{97}\right)$ da altura, peso, IMC e PC por idade e sexo.

\begin{tabular}{|c|c|c|c|c|c|c|c|c|c|c|c|c|c|c|}
\hline \multirow{3}{*}{ Idade } & \multicolumn{7}{|c|}{ Masculino } & \multicolumn{7}{|c|}{ Feminino } \\
\hline & \multicolumn{14}{|c|}{ Altura $(\mathrm{cm})$} \\
\hline & P3 & P10 & P25 & P50 & P75 & P90 & P97 & P3 & P10 & P25 & P50 & P75 & P90 & P97 \\
\hline 6 & 109,42 & 112,37 & 115,40 & 118,51 & 121,71 & 125,01 & 128,39 & 108,95 & 111,56 & 114,24 & 117,00 & 119,85 & 122,77 & 125,79 \\
\hline 7 & 113,43 & 116,56 & 119,79 & 123,13 & 126,58 & 130,15 & 133,83 & 112,91 & 115,85 & 118,88 & 122,01 & 125,24 & 128,56 & 132,00 \\
\hline 8 & 118,57 & 121,88 & 125,32 & 128,90 & 132,63 & 136,50 & 140,54 & 117,87 & 121,12 & 124,51 & 128,03 & 131,70 & 135,52 & 139,51 \\
\hline 9 & 123,53 & 127,05 & 130,72 & 134,55 & 138,54 & 142,71 & 147,06 & 122,80 & 126,20 & 129,83 & 133,69 & 137,82 & 142,24 & 146,98 \\
\hline \multirow[t]{3}{*}{10} & 127,99 & 131,72 & 135,60 & 139,66 & 143,91 & 148,36 & 153,02 & 129,02 & 132,47 & 136,22 & 140,32 & 144,83 & 149,81 & 155,37 \\
\hline & \multicolumn{14}{|c|}{ Peso $(\mathrm{kg})$} \\
\hline & P3 & P10 & P25 & P50 & P75 & P90 & P97 & P3 & P10 & P25 & P50 & P75 & P90 & P97 \\
\hline 6 & 17,85 & 19,33 & 21,00 & 22,92 & 25,13 & 27,70 & 30,73 & 17,18 & 18,64 & 20,32 & 22,27 & 24,57 & 27,31 & 30,61 \\
\hline 7 & 19,28 & 21,01 & 23,04 & 25,46 & 28,36 & 31,93 & 36,38 & 18,69 & 20,41 & 22,44 & 24,89 & 27,88 & 31,61 & 36,38 \\
\hline 8 & 21,31 & 23,38 & 25,89 & 28,97 & 32,84 & 37,87 & 44,62 & 20,98 & 23,05 & 25,54 & 28,63 & 32,52 & 37,58 & 44,43 \\
\hline 9 & 23,31 & 25,77 & 28,77 & 32,51 & 37,26 & 43,52 & 52,09 & 23,08 & 25,49 & 28,43 & 32,09 & 36,76 & 42,90 & 51,33 \\
\hline \multirow[t]{3}{*}{10} & 25,42 & 28,28 & 31,76 & 36,06 & 41,49 & 48,52 & 57,93 & 26,41 & 29,24 & 32,72 & 37,07 & 42,68 & 50,18 & 60,68 \\
\hline & \multicolumn{14}{|c|}{$\operatorname{IMC}\left(\mathrm{kg} / \mathrm{m}^{2}\right)$} \\
\hline & P3 & P10 & P25 & P50 & P75 & P90 & P97 & P3 & P10 & P25 & P50 & P75 & P90 & P97 \\
\hline 6 & 14,02 & 14,60 & 15,26 & 16,00 & 16,84 & 17,83 & 18,99 & 13,45 & 14,23 & 15,12 & 16,12 & 17,28 & 18,62 & 20,20 \\
\hline 7 & 14,03 & 14,75 & 15,58 & 16,56 & 17,73 & 19,17 & 20,98 & 13,77 & 14,61 & 15,59 & 16,74 & 18,10 & 19,77 & 21,84 \\
\hline 8 & 14,22 & 15,07 & 16,09 & 17,32 & 18,86 & 20,86 & 23,59 & 14,06 & 14,98 & 16,07 & 17,39 & 19,03 & 21,13 & 23,95 \\
\hline 9 & 14,42 & 15,36 & 16,50 & 17,90 & 19,71 & 22,15 & 25,68 & 14,26 & 15,28 & 16,51 & 18,01 & 19,93 & 22,44 & 25,94 \\
\hline \multirow[t]{3}{*}{10} & 14,90 & 15,90 & 17,11 & 18,62 & 20,57 & 23,24 & 27,18 & 14,45 & 15,58 & 16,96 & 18,67 & 20,87 & 23,81 & 27,98 \\
\hline & \multicolumn{14}{|c|}{$\mathrm{PC}(\mathrm{cm})$} \\
\hline & P3 & P10 & P25 & P50 & P75 & P90 & P97 & P3 & P10 & P25 & P50 & P75 & P90 & P97 \\
\hline 6 & 49,36 & 50,82 & 52,46 & 54,34 & 56,53 & 59,10 & 62,23 & 48,78 & 50,31 & 52,00 & 53,88 & 56,00 & 58,40 & 61,17 \\
\hline 7 & 50,16 & 51,97 & 54,08 & 56,62 & 59,74 & 63,75 & 69,22 & 48,94 & 50,93 & 53,16 & 55,69 & 58,60 & 61,99 & 66,00 \\
\hline 8 & 51,68 & 53,93 & 56,63 & 59,98 & 64,32 & 70,30 & 79,45 & 49,90 & 52,42 & 55,29 & 58,59 & 62,42 & 66,94 & 72,37 \\
\hline 9 & 53,31 & 56,10 & 59,45 & 63,56 & 68,81 & 75,85 & 86,06 & 51,55 & 54,60 & 58,07 & 62,03 & 66,63 & 72,01 & 78,42 \\
\hline 10 & 54,31 & 57,51 & 61,30 & 65,91 & 71,68 & 79,16 & 89,41 & 52,96 & 56,38 & 60,23 & 64,57 & 69,50 & 75,16 & 81,69 \\
\hline
\end{tabular}

Rev. bras. Educ. Fís. Esporte, São Paulo, v.26, n.4, p.611-25, out./dez. 2012 • 615 

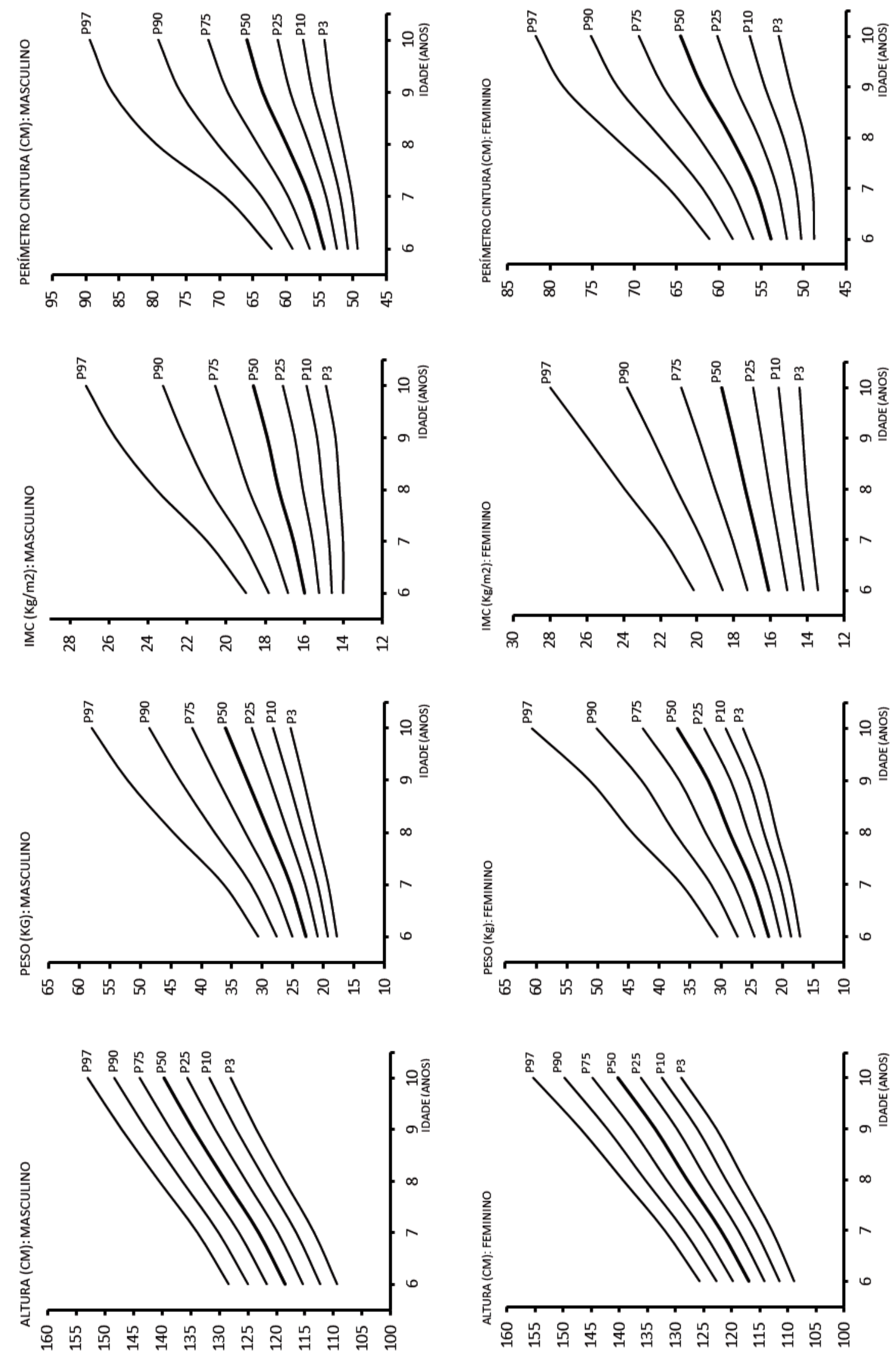

FIGURA 1 - Cartas percentílicas de referência para a altura, peso, IMC e PC (masculino e feminino). 
TABELA 3 -Valores médios ( \pm desvio-padrão) da Maia, valores do Percentil 50 do CDC, diferença de médias e valor de prova (p) para a altura, peso e IMC de meninos e meninas.

\begin{tabular}{|c|c|c|c|c|c|c|c|c|}
\hline \multicolumn{5}{|c|}{ Masculino } & \multicolumn{4}{|c|}{ Feminino } \\
\hline \multirow[t]{2}{*}{ Idade } & $\begin{array}{c}\text { Maia } \\
M \pm \mathrm{DP}\end{array}$ & $\begin{array}{l}\text { CDC } \\
\text { P } 50\end{array}$ & $\begin{array}{l}\text { Diferença } \\
\text { de médias }\end{array}$ & $p$ & $\begin{array}{c}\text { Maia } \\
M \pm \mathrm{DP}\end{array}$ & $\begin{array}{l}\text { CDC } \\
\text { P } 50\end{array}$ & $\begin{array}{l}\text { Diferença } \\
\text { de médias }\end{array}$ & $p$ \\
\hline & \multicolumn{8}{|c|}{ Altura } \\
\hline $6+$ & $121,3 \pm 5,1$ & 118,6 & 2,8 & $<0,001$ & $119,9 \pm 4,6$ & 117,5 & 2,4 & $<0,001$ \\
\hline $7+$ & $126,0 \pm 5,7$ & 125,0 & 1,1 & $<0,01$ & $125,3 \pm 5,6$ & 123,6 & 1,7 & $<0,001$ \\
\hline $8+$ & $132,2 \pm 5,8$ & 130,2 & 2,0 & $<0,001$ & $131,0 \pm 5,9$ & 130,3 & 0,8 & $<0,004$ \\
\hline $9+$ & $137,3 \pm 6,3$ & 135,9 & 1,4 & $<0,001$ & $137,5 \pm 6,9$ & 135,5 & 2,0 & $<0,001$ \\
\hline \multirow[t]{2}{*}{$10+$} & $141,0 \pm 6,4$ & 141,2 & $-0,2$ & $<0,660$ & $140,8 \pm 7,1$ & 141,2 & $-0,3$ & $<0,584$ \\
\hline & \multicolumn{8}{|c|}{ Peso } \\
\hline $6+$ & $24,9 \pm 3,8$ & 21,7 & 3,2 & $<0,001$ & $24,1 \pm 3,6$ & 21,1 & 3,0 & $<0,001$ \\
\hline $7+$ & $27,9 \pm 5,3$ & 24,6 & 3,3 & $<0,001$ & $27,6 \pm 5,4$ & 23,6 & 4,0 & $<0,001$ \\
\hline $8+$ & $32,0 \pm 6,7$ & 26,7 & 5,3 & $<0,001$ & $31,2 \pm 6,2$ & 26,8 & 4,5 & $<0,001$ \\
\hline $9+$ & $35,7 \pm 7,5$ & 30,1 & 5,6 & $<0,001$ & $35,8 \pm 7,5$ & 30,1 & 5,7 & $<0,001$ \\
\hline \multirow[t]{2}{*}{$10+$} & $37,8 \pm 8,2$ & 33,5 & 4,3 & $<0,001$ & $38,0 \pm 7,0$ & 33,6 & 4,4 & $<0,001$ \\
\hline & \multicolumn{8}{|c|}{ IMC } \\
\hline $6+$ & $16,6 \pm 1,6$ & 15,4 & 1,1 & $<0,001$ & $16,7 \pm 1,7$ & 15,2 & 1,5 & $<0,001$ \\
\hline $7+$ & $17,2 \pm 2,1$ & 15,6 & 1,6 & $<0,001$ & $17,5 \pm 2,4$ & 15,4 & 2,1 & $<0,001$ \\
\hline $8+$ & $18,1 \pm 2,6$ & 15,9 & 2,2 & $<0,001$ & $18,1 \pm 2,7$ & 15,8 & 2,3 & $<0,001$ \\
\hline $9+$ & $18,8 \pm 2,9$ & 16,2 & 2,6 & $<0,001$ & $19,0 \pm 3,1$ & 16,4 & 2,7 & $<0,001$ \\
\hline $10+$ & $19,1 \pm 2,7$ & 16,9 & 2,1 & $<0,001$ & $19,0 \pm 2,9$ & 16,9 & 2,1 & $<0,001$ \\
\hline
\end{tabular}

Na FIGURA 2 é apresentada a comparação dos valores do $\left(\mathrm{P}_{50}\right)$ da altura, peso e IMC das crianças maiatas com os de outros estudos portugueses. As meninas maiatas aos seis anos de idade são mais baixas que as meninas de Amarante e de Viana do Castelo mas mais altas que as açorianas e madeirenses. Porém, nas meninas maiatas verifica-se que a diferença de altura dos nove para os 10 anos é de $+6,63 \mathrm{~cm}$, muito superior a todas as demais, de tal forma que nesta idade (10 anos) são as mais altas. Os meninos, tal como as meninas, são aos seis anos mais baixos em relação aos Amarantinos e Vianenses, situação que se mantém aos 10 anos de idade. No peso, as meninas da Maia apresentam, aos 10 anos, valores superiores relativamente a todos as outras, sendo que a diferença se situa entre $+2,07 \mathrm{~kg} \mathrm{a}+2,97 \mathrm{~kg}$. A diferença nos meninos é um pouco menor já que varia no mesmo escalão etário entre $+1,46 \mathrm{~kg} \mathrm{e}+2,06 \mathrm{~kg}$. No IMC, em todas as idades e nos dois sexos, os valores das crianças maiatas são sempre mais elevados, com exceção dos seis e sete anos em que as crianças açorianas têm valores ligeiramente superiores. 

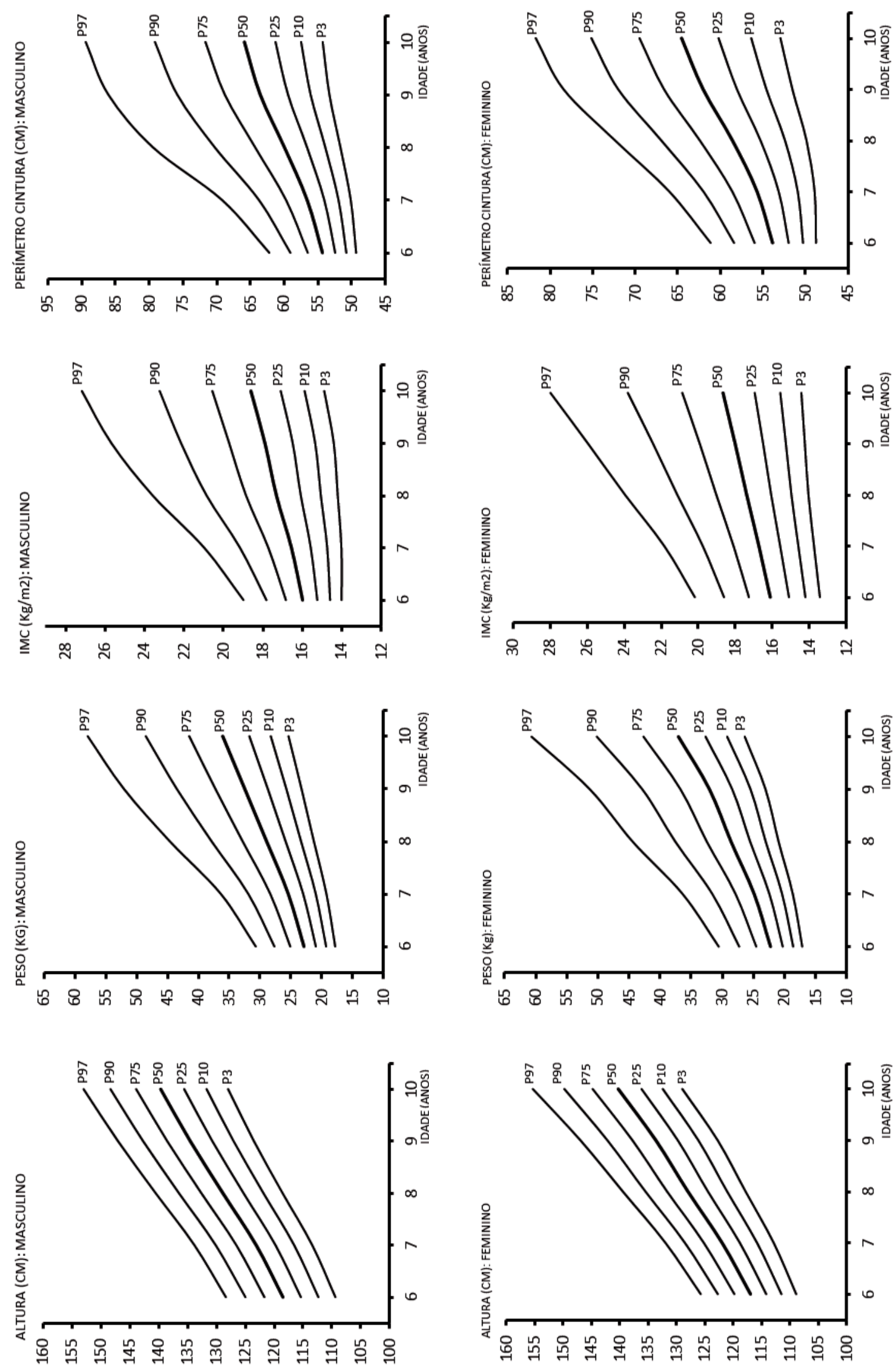

FIGURA 2 -Comparação dos valores do $\left(\mathrm{P}_{50}\right)$ da altura, peso e IMC com os de outros estudos portugueses (masculino e feminino).

618 • Rev. bras. Educ. Fís. Esporte, São Paulo, v.26, n.4, p.611-25, out./dez. 2012 


\section{Discussão}

O presente estudo apresenta valores de referência e cartas percentílicas para a altura, peso, IMC e PC para crianças, do Concelho da Maia, em Portugal, bem como contrasta os resultados encontrados com uma referência internacional (CDC) e com os observados em outras investigações realizadas em Portugal.

$\mathrm{O}$ valor e significado dos resultados obtidos dos modelos construídos com o método LMS estão dependentes, em grande medida, da qualidade da informação demonstrada na reduzida variação de medidas sucessivas, i.e., na precisão dos valores da altura, peso e PC, dado estarem nos limites de tolerância aceitáveis para estas variáveis, respetivamente de 0,2 cm, 0,2 kg e $3 \mathrm{~cm}$ (LoHman, Roche $\&$ Martorell, 1988). Um outro aspecto importante refere-se à elevada dimensão amostral entre sexos e idades fato que permite obter estimativas mais precisas e robustas dos percentis (ver resultados do ANEXO 1). De igual modo, tal como referem outros estudos (PAn \& Cole, 2004; Royston \& Wright, 2000; Van Buuren \& FredriKs, 2001) também as representações gráficas do "Q-test" e "Worm-plots" mostraram-se ferramentas úteis na verificação da qualidade do ajustamento dos modelos, permitindo selecionar o mais adequado. Os graus de liberdade dos modelos considerados são semelhantes aos limites estabelecidos como adequados por PAN e COLE (2011) em função da dimensão amostral bem como na escolha da escala para a idade.

$\mathrm{Na}$ trajetória modal $\left(\mathrm{P}_{50}\right)$ da altura e peso os meninos de seis anos de idade são mais altos e pesados que as meninas $(+1,51 \mathrm{~cm} \mathrm{e}+0,64 \mathrm{~kg})$, situação que se vai dissipando com a idade, de tal forma que aos 10 anos as meninas invertem esta posição, passando a ser mais altas e pesadas $(+0,65 \mathrm{~cm} \mathrm{e}+1,01 \mathrm{~kg})$. Este comportamento dos resultados é semelhante ao de outras pesquisas realizadas no Brasil (GUEDEs, DE Matos, Lopes, Ferreirinha \& Silva, 2010; Silva et al., 2011), Turquia (Neyzi, Furman, BundaK, GunOZ, DARENDELILER \& BAS, 2006), Itália (CACCIARI, Milani, Balsamo, Dammacco, De Luca, Chiarelli, Pasquino, Tonini \& Vanelli, 2002) e México (Malina, Zavaleta \& Little, 1987), sugerindo o início do "take-off" do salto pubertário que ocorre aproximadamente dois anos mais cedo no sexo feminino (Malina, Bouchard \& Beunen, 1988).

No IMC são expectáveis aumentos em ambos os sexos ao longo da idade. Nas trajetórias do $\mathrm{P}_{3}$ e $\mathrm{P}_{97}$ verifica-se um distanciamento cada vez maior entre os seis e os 10 anos de idade, sendo essa diferença no $\mathrm{P}_{3} \mathrm{e}$ no $\mathrm{P}_{97}$ respetivamente para os meninos $+0,88 \mathrm{~kg} / \mathrm{m}^{2} \mathrm{e}$ $+8,19 \mathrm{~kg} / \mathrm{m}^{2}$; e para as meninas, $+1,0 \mathrm{~kg} / \mathrm{m}^{2} \mathrm{e}+7,79$ $\mathrm{kg} / \mathrm{m}^{2}$. Não obstante ser um indicador indireto do estado nutricional, o IMC é amplamente utilizado para classificar, com base em valores de corte consensuais, excesso de peso e obesidade em crianças e adultos, pelo que a monitorização dos seus valores e alteraçóes induzidas pela idade e múltiplos fatores ambientais é fundamental. Ora crianças com sobrepeso e obesas têm seis vezes mais chances de se tornarem adultos com sobrepeso e/ou obesos, a que se associam riscos de outras co-morbilidades (Herman, Craig, Gauvin \& KatZMARZYK, 2009; JuHOLA et al., 2011). Por sua vez, o PC é considerado como o melhor indicador da obesidade central relacionada com fatores de risco em adultos, pelo que a sua utilização vem sendo cada vez mais valorizada (ARDERn, JANSSEn, Ross \& KatZMARZyK, 2004).

A comparação dos resultados do PC com os de outros estudos não é tarefa fácil, já que não existem referências internacionais unanimemente aceites, do mesmo modo que não existe consenso acerca do local ideal para a sua medição (Ross, BERENTZEN, Bradshaw, Janssen, Kahn, Katzmarzyk, KuK, Seidell, Snijder, Sorensen \& Despres, 2008). Os seus resultados têm um comportamento semelhante aos do IMC, observando-se também, quando avançamos do $\mathrm{P}_{3}$ para o $\mathrm{P}_{97}$, uma amplitude de valores cada vez maior entre os seis e os 10 anos, sendo essa diferença no $\mathrm{P}_{3}$ e no $\mathrm{P}_{97}$ respetivamente para os meninos $+4,95 \mathrm{~cm} \mathrm{e}+27,19 \mathrm{~cm}$ e para as meninas, $+4,18 \mathrm{~cm} \mathrm{e}+20,52 \mathrm{~cm}$, o que nos leva a pensar a existência de uma relação estreita entre estas medidas. A FIGURA 3 representa trajetórias modais do $\mathrm{PC}$ das crianças maiatas em relação às australianas (EISENMANN, 2005), turcas (HATIPOGLU, Ozturk, Mazicioglu, Kurtoglu, Seyhan \& LoKoGlu, 2008), chinesas (Liu, Hills, Hu, Li, Du, Xu, Byrne \& MA, 2010) e inglesas (McCARThy, JARRET \& Crawley, 2001). As crianças da Maia apresentam valores mais elevados, sendo que nas meninas a discrepância é acentuada a partir dos sete anos de idade. Convém ter presente que nos diferentes países o local anatômico de medição é distinto, nas crianças australianas foi medido ao nível do umbigo e nas turcas, chinesas e inglesas foi medido no ponto médio entre o bordo da última costela e o topo da crista ilíaca. Contudo, mesmo considerando estas diferenças e tomando para comparação as crianças que se encontram mais próximas (chinesas) e as que se encontram mais afastadas (inglesas) das maiatas, 
torna-se evidente que aos 10 anos de idade o distanciamento das crianças da Maia é maior no sexo

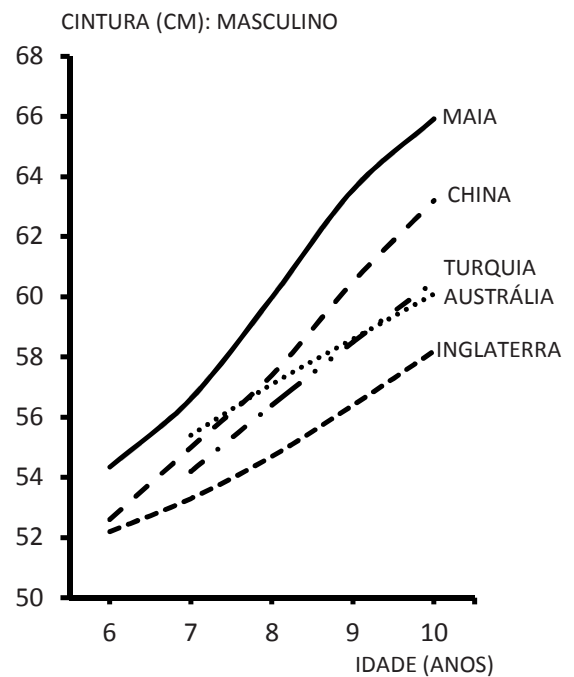

feminino do que no masculino (meninos $2,71 \mathrm{~cm}$ e 7,71 cm; e meninas $5,27 \mathrm{~cm} \mathrm{e} \mathrm{7,87} \mathrm{cm).}$

CINTURA (CM): FEMININO

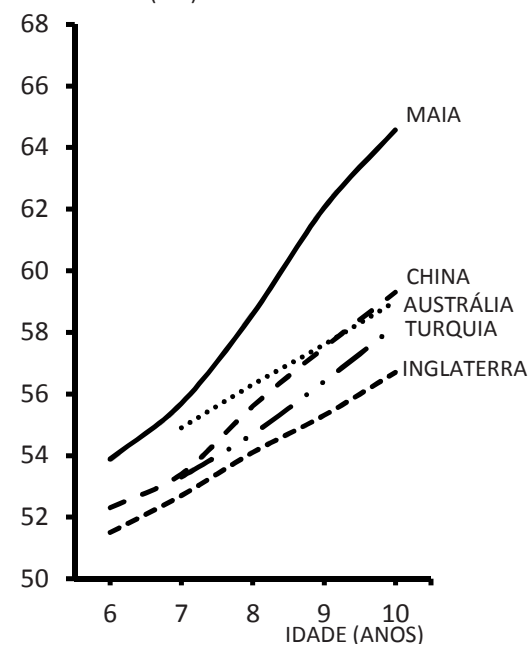

FIGURA 3 -Comparação dos valores do $\mathrm{P}_{50}$ do PC com os outros estudos (masculino e feminino).

Dos resultados do QUADRO 3 ficou claro que as crianças da Maia, dos seis aos nove anos de idade, são significativamente mais altas $(\mathrm{p}<0,01)$ que as do CDC. Aos 10 anos de idade as diferenças estaturais não têm qualquer significado estatístico. Este mesmo padrão é observado com outras crianças portuguesas (resultados não apresentados) das cidades de Amarante (Sousa \& Maia, 2005) e de Viana de Castelo (RodriGUeS, Bezerra \& Saraiva, 2007). Estas diferenças são difíceis de explicar embora alguma argumentação esteja centrada em distintos aspectos sociogeográficos, que privilegia condições de vida, nutrição, saúde e fatores econômicos (BIELICKI, 1986; ULIJASZEK, 2006).

Em relação ao peso e ao IMC as crianças da Maia, em todas as idades são significativamente mais pesadas e com IMC mais elevados $(\mathrm{p}<0,001)$ que as do CDC. Aos seis e 10 anos de idade as meninas e meninos pesam mais $3 \mathrm{~kg}$ e 4,4 kg; e 3,2 kg e 4,3 $\mathrm{kg}$, respetivamente aos do CDC. Quanto ao IMC, aos seis anos de idade, por exemplo, as meninas e meninos maiatos têm valores superiores em $1,5 \mathrm{~kg} /$ $\mathrm{m}^{2}$ e $1,1 \mathrm{~kg} / \mathrm{m}^{2}$, respetivamente, e aos 10 anos tanto meninas como os meninos apresentam valores de $2,1 \mathrm{~kg} / \mathrm{m}^{2}$ superiores aos do CDC. Estas diferenças são concordantes com dados de estudos realizados nas cidades de Viana do Castelo, Amarante e nas Regiōes Autônomas da Madeira e dos Açores. Decorre daqui um padrão relativamente mais longilíneo na forma e tamanho do grupo das crianças do CDC e é provável, ainda que não haja informação factual, que distintos hábitos alimentares possam justificar tal diferença.
Quando comparamos os valores do crescimento de diferentes locais e regiōes, intra país (i.e. Portugal), importa referir alguns problemas: 1) o desfasamento temporal dos estudos, embora em nenhum deles tenha o efeito de tendência decenal; 2) os protocolos utilizados; 3) a dimensão amostral e; 4) a estratificação etária. Os estudos portugueses são provenientes de sub-regiōes distintas cuja estrutura socioeconômica não é substancialmente diferenciada. Um aspecto importante a reter na comparação destes resultados decorre das lições do teorema do limite central, em estatística, sobretudo no que diz respeito à distribuição das médias amostrais. Não é de esperar que as médias da altura, peso, IMC e PC sejam iguais entre locais e regióes por questões de erros amostrais (não confundir com o processo de amostragem em cada local/região) que implicam variabilidade nas médias que pode ter, ou não, uma qualquer explicação que não a mera casualidade.

As crianças maiatas têm uma estatura média muito similar às madeirenses, mas inferior às crianças amarantinas e vianenses, com exceção das meninas aos 10 anos e superior às açorianas. A altura é uma variável sensível à influência de fatores ambientais, econômicos e sociais. Numa tentativa de encontrarmos uma justificação para estes resultados e colocando desde logo de parte o acesso aos cuidados primários de saúde, já que em Portugal o Sistema Nacional de Saúde é gratuito para as crianças, procuramos encontrar esta justificação num eventual gradiente econômico. Utilizando o "ranking" do Índice Concelhio de Desenvolvimento Econômico e Social (ICDES) dos 278 
Concelhos do Continente (MANSO \& SiMŌES, 2009) em que a Maia se encontra em (38 lugar $/ \mathrm{ICDES}=$ 77,3 ), seguida de Viana de Castelo (82० lugar/ICDES $=69,9)$ e depois de Amarante (197\% lugar $/$ ICDES = 57,3 ), e ao hierarquizarmos os valores da altura em função do mesmo, também aqui não encontramos espaço de justificação.

No peso, as crianças maiatas aos 10 anos de idade são as mais pesadas. Essas diferenças podem ser justificadas nas meninas com 10 anos, pelo já alegado início do salto pubertário. Frequentemente se associa o desenvolvimento econômico de uma cidade ao acesso a alimentos de baixo preço, muitas vezes de alto teor calórico, que poderão ajudar a justificar a presença destes valores tão elevados do peso nas crianças maiatas.

No IMC, e a partir dos sete anos, as crianças maiatas apresentam valores superiores. A amplitude do IMC dos seis para os 10 anos de idade é igual nos dois sexos $\left(2,6 \mathrm{~kg} / \mathrm{m}^{2}\right)$ e superior quando em comparação com os outros estudos portugueses, que apresentam os seguintes valores para as meninas: Madeira $\left(1,5 \mathrm{~kg} / \mathrm{m}^{2}\right)$, Açores e Amarante $\left(1,6 \mathrm{~kg} / \mathrm{m}^{2}\right)$; e para os meninos: Amarante $\left(1,5 \mathrm{~kg} / \mathrm{m}^{2}\right)$, Açores $\left(1,6 \mathrm{~kg} / \mathrm{m}^{2}\right)$ e Madeira $(2,0 \mathrm{~kg} /$ $\mathrm{m}^{2}$ ). Estes resultados exigem um cuidado particular na sua monitorização e vigilância face à necessidade em elaborar, desde cedo, estratégias eficazes no seu combate.

O presente estudo tem algumas limitações que passamos a destacar: 1) o delineamento transversal não permitiu a realização de inferências sobre mudanças intraindividuais ao longo da idade e; 2) a ausência de informação do estatuto socioeconômico embora não seja claro o seu valor interpretativo face a alguma semelhança nas condições econômicas dos locais de amostragem das crianças maiatas.

Contudo, esta pesquisa é portadora de alguns pontos fortes que consideramos importantes: 1) a magnitude da dimensão da amostra e sua representatividade local; 2) o uso de ferramentas sofisticadas de análise estatística e de representação gráfica da qualidade dos modelos; 3) a relevância de valores de referência da altura, peso, IMC e PC num concelho que não possuía esta informação, cujo valor é indesmentível para pediatras e outros profissionais de saúde, investigadores, comunidade educativa, encarregados e educação e poder político local; 4) a apresentação dos primeiros valores de referência do $\mathrm{PC}$ de crianças dos seis aos 10 anos de idade em Portugal.

Em conclusão, a altura, peso, IMC e PC das crianças maiatas aumentam linearmente com a idade, sendo que aos 10 anos as meninas são mais altas e pesadas do que os meninos. A altura média das meninas e meninosmaiatos, dos seis aos nove anos, é significativamente superior às do CDC mas, aos 10 anos, não existem diferenças com qualquer significado estatístico. O peso e o IMC das crianças maiatas são superiores às referências do CDC. As trajetórias modais do PC das crianças maiatas são superiores em relação às australianas, turcas, chinesas e inglesas. Observam-se diferenças nas variáveis somáticas em locais e regiōes distintas de Portugal.

\begin{abstract}
Growth reference charts of six to 10 year old children from the Maia County, Portugal

The purposes of this study were to construct height, weight, body mass index (BMI) and waist circumference (WC) percentile charts of Maia county children, Portugal; to compare Maia county data with reference data from other Portuguese studies and from Center for Disease Control and Prevention (CDC). The sample comprises 3749 children (1942 boys and 1807 girls) aged six to 10 years. Charts were separately built for each sex using the LMS method. Maia children's height, weight, BMI and WC increase with age in linear and nonlinear fashions. Maia children showed consistently higher values in height, weight and BMI than the CDC references. WC modal trajectories of Maia Children are higher than other international studies. Differences in somatic variables are observed in distinctive locals and regions of Portugal.
\end{abstract}

UnITERMS: Reference charts; Height; Weight; BMI; Waist circumference. 


\section{Resumen}

Cartas de referencia del crecimiento somático de niños de seis a los 10 años de edad del Concejo de Maia, Portugal

Los propósitos del estudio fueron construir cartas percentílicas para la altura, peso, índice de masa corporal (IMC) y perimetro de la cintura (PC) de los escolares del Concejo de Maia, Portugal; contrastar los resultados encontrados en el Concejo de Maia con otros estudios realizados en Portugal y con las referencias del Center for Disease Control and Prevention (CDC). La muestra está constituída por 3749 escolares (1942 niños y 1807 niñas) de seis a los 10 años de edad. Las cartas fueron construídas por separado para cada sexo utilizando el método LMS. La altura, peso, IMC y PC de los escolares Maiatas aumentan de modo lineal y no lineal con la edad. Los Maiatos muestran valores medios de altura, peso y IMC consistentemente superiores a los del CDC. Las trayectorias modales del PC de los Maiatos son superiores a los estudios internacionales. Se observan diferencias en las variables somáticas en localidades y regiones distintas de Portugal.

Palabras clave: Cartas percentílicas; Altura; Peso; IMC; Perímetro de la cintura.

\section{Referências}

ALBERTI, K.; ZIMMET, P.; SHAW, J. Metabolic syndrome:a new world-wide definition: a consensus statement from the international diabetes federation. Diabet Medicine, Chichester, v.23, p.469-80, 2006.

ARDERN, C.I.; JANSSEN, I.; ROSS, R.; KATZMARZYK, P.T. Development of health-related waist circumference thresholds within BMI categories. Obesity Research, Baton Rouge, v.12, n.7, p.1094-103, 2004.

BIELICKI, T. Physical growth as a measure of the rconomic well-being of populations: the twentieth century. In: FALKNER, F.; TANNER, J. (Eds.). Human growth: a compreensive treatise. New York: Plenum Press, 1986. p.283-305.

CACCIARI, E.; MILANI, S.; BALSAMO, A.; DAMMACCO, F.; DE LUCA, F.; CHIARELLI, F.; PASQUINO, A.M.; TONINI, G.; VANELLI, M. Italian cross-sectional growth charts for height, weight and BMI (6-20 y). European Journal of Clinical Nutrition, London, v.56, n.2, p.171-80, 2002.

CENTER FOR DISEASE CONTROL AND PREVENTION (CDC). Prevalence of overweight among children and adolescents: United States. 2006. Disponível em: <http://www.cdc.gov/nchs/pressroom/06facts/obesity03_04.htm>.

COLE, T.J.; FREEMAN, J.V.; PREECE, M.A. British 1990 growth reference centiles for weight, height, body mass index and head circumference fitted by maximum penalized likelihood. Statistics in Medicine, Chichester, v.17, n.4, p.407-29, 1998. COLE, T.J.; GREEN, P.J. Smoothing reference centile curves: the LMS method and penalized likelihood. Statistics in Medicine, Chichester, v.11, n.10, p.1305-19, 1992.

DE ONIS, M.; BLOSSNER, M.; BORGHI, E. Global prevalence and trends of overweight and obesity among preschool children. The American Journal of Clinical Nutrition, Bethesda, v.92, n.5, p.1257-64, 2010.

DE ONIS, M.; GARZA, C.; VICTORA, C.G.; BHAN, M.K.; NORUM, K.R. The WHO multicentre growth reference study (MGRS): rationale, planning and implementation. Food and Nutrition Bulletin, Tokyo, v.25 n.1, p.S3-S4, 2004. DE ONIS, M.; ONYANGO, A.W.; BORGHI, E.; SIYAM, A.; NISHIDA, C.; SIEKMANN, J. Development of a WHO growth reference for school-aged children and adolescents. Bulletin of the World Health Organization, Geneva, v.85, n.9, p.660-67, 2007. EISENMANN, J.C. Waist circumference percentiles for 7- to 15-year-old Australian children. Acta Paediatrica, Oslo, v.94, n.9, p.1182-85, 2005.

EVELETH, P.B.; TANNER, J.M. Worldwide variation in human growth. 2nd. ed. Cambridge: Cambridge University Press, 1990. FREITAS, D.; MAIA, J.; BEUNEN, G.; CLAESSENS, A.; THOMIS, M.; MARQUES, A.; RODRIGUES, A.; SILVA, C.; CRESPO, M. Crescimento somático, maturação biológica, aptidão física, actividade física e estatuto sócio-económico de crianças e adolescentes madeirenses: o estudo de crescimento da Madeira. Funchal: Universidade da Madeira, 2002. GOLDSTEIN, H.; TANNER, J.M. Ecological considerations in the creation and the use of child growth standards. Lancet, New York, v.1, n.15, p.582-84, 1980.

GUEDES, D.P.; DE MATOS, J.A.; LOPES, V.P.; FERREIRINHA, J.E.; SILVA, A.J. Physical growth of schoolchildren from the Jequitinhonha Valley, Minas Gerais, Brazil: comparison with the CDC-2000 reference using the LMS method. Annals of Human Biology, London, v.37, n.4, p.574-84, 2010. 
HATIPOGLU, N.; OZTURK, O.; MAZICIOGLU, M.M.; KURTOGLU, S.; SEYHAN, S.; LOKOGLU, F. Waist circumference percentiles for 7- to 17-year-old Turkish children and adolescents. European Journal of Pediatrics, Berlin, v.167, p.383-89, 2008.

HERMAN, K.M.; CRAIG, C.L.; GAUVIN, L.; KATZMARZYK, P.T. Tracking of obesity and physical activity from childhood to adulthood: the physical activity longitudinal study. International Journal of Pediatric Obesity, Colchester, v.4, p.281-88, 2009. INSTITUTO NACIONAL DE ESTATÍSTICA, IP (INE). Anuário estatístico da região norte 2008. Lisboa: INE, 2009. Anuário estatístico da região norte 2010. Lisboa: INE, 2011.

INTERNATIONAL OBESITYTASK FORCE (IOTF). Obesity in Europe. Brussels: European Association for the Study of Obesity, 2005. (International Obesity Task Force EU Platform Briefing Paper).

JANSSEN, I.; KATZMARZYK, P.T.; ROSS, R. Waist circumference and not body mass index explains obesity-related health risk. The American Journal of Clinical Nutrition, Bethesda, v.79, n.3, p.379-84, 2004.

JOHNSTON, F; BOGIN, B.; MacVEAN, R.; NEWMAN, B. A comparison of International Standards versus local reference data for the triceps and subscapular skinfolds of Guatemalan children and youth. Human Biology, Detroit, v.56, n.1, p.157-71, 1984 .

JUHOLA, J.; MAGNUSSEN, C.G.; VIIKARI, J.S.; KÄHÖNEN, M.; HUTRI-KÄHÖNEN, N.; JULA, A.; LEHTIMÄKI, T.; AKERBLOM, H.K.; PIETIKÄINEN, M.; LAITINEN, T.; JOKINEN, E.; TAITTONEN, L.; RAITAKARI, O.T.; JUONALA, M. Tracking of serum lipid levels, blood pressure, and body mass index from childhood to adulthood: the cardiovascular risk in young Finns Study. The Journal of Pediatrics, Saint Louis, v.159, n.4, p.584-90, 2011.

LIU, A.; HILLS, A.P.; HU, X.; LI, Y.; DU, L.; XU, Y.; BYRNE, N.M.; MA, G. Waist circumference cut-off values for the prediction of cardiovascular risk factors clustering in Chinese school-aged children: a cross-sectional study. BMC Public Health, London, v.10, p.82, 2010.

LOFREN, I.; HERRON, K.; ZERN, T.; WEST, K.; PATALAY, M.; SHACHTER, N.S.; KOO, S.I.; FERNANDEZ, M.L. Waist circumference is a better predictor than body mass index for coronary heart disease risk in overweight premenopausal women. Journal of Nutrition, Rockville, v.134, p.1071-76, 2004.

LOHMAN, T.G.; ROCHE, A.F.; MARTORELL, R. Anthropometric standardization reference manual. Champaign: Human Kinetics, 1988.

MAIA, J.A.R.; LOPES, V.P. Estudo do crescimento somático, aptidão física, actividade física e capacidade de coordenação corporal de crianças do $1^{\circ}$ ciclo do ensino básico da região Autónoma dos Açores. Ponta Delgada: Região Autónoma dos Açores/Faculdade de Desporto da Universidade do Porto, 2002.

Crescimento e desenvolvimento de crianças e jovens açorianos: o que pais, professores, pediatras e nutricionistas gostariam de saber. Ponta Delgada: Região Autónoma dos Açores/Faculdade de Desporto da Universidade do Porto, 2007. MALINA, R.M.; BOUCHARD, C.; BEUNEN, G. Human growth: selected aspects of current research on well-nourished children. Ann Rev Anthropol, v.17, p.187-219, 1988.

MALINA, R.M.; ZAVALETA, A.N.; LITTLE, B.B. Body size, fatness, and leanness of Mexican American children in Brownsville, Texas: changes between 1972 and 1983. American Journal of Public Health, New York, v.77, n. 5, p. 573-77, 1987. MANSO, J.R.P.; SIMÕES, N.M. Indicador sintético de desenvolvimento económico e social ou de bem-estar dos municípios do continente português. Santa Maria: Universidade da Beira Interior, 2009. (Observatório para o Desenvolvimento Económico e Social).

McCARTHY, H.D.; JARRET, K.V.; CRAWLEY, H.F. The development of waist circumference percentiles in British children aged $5.0 \pm 16.9$ y. European Journal of Clinical Nutrition, London, v.55, p.902-07, 2001.

NEYZI, O.; FURMAN, A.; BUNDAK, R.; GUNOZ, H.; DARENDELILER, F.; BAS, F. Growth references for Turkish children aged 6 to 18 years. Acta Paediatrica, Oslo, v.95, p.1635-41, 2006.

PAN, H.; COLE, T.J. A comparison of goodness of fit tests for age-related reference ranges. Statistics in Medicine, Chichester, v.23, n.11, p.1749-65, 2004.

LmsChartMaker: a program to construct growth references using the LMS method. Version 2.45. 2011. Disponível em: <http://www.healthforallchildren.co.uk>.

PEREIRA, A.M.R. Crescimento somático e aptidão física de crianças com idades compreendidas entre os $\mathbf{6}$ e os 10 anos de idade: um estudo no Concelho da Maia. Porto: Faculdade de Ciências do Desporto e Educação Física/Universidade do Porto, 2000. PEREIRA, S.A.; SEABRA, A.T.; SILVA, R.G.; KATZMARZYK, P.T.; BEUNEN, G.P.; MAIA, J.A. Prevalence of overweight, obesity and physical activity levels in children from Azores Islands. Annals of Human Biology, London, v.37, n. 5, p.682-91, 2010. RODRIGUES, L.P.; BEZERRA, P.; SARAIVA, L. Morfologia e crescimento dos 6 aos 10 anos de idade em Viana do Castelo, Portugal. Revista Motricidade, Vila Real, v.3, n.4, p.55-75, 2007. 
RORIZ, M.S. et al.

ROSS, R.; BERENTZEN, T.; BRADSHAW, A.J.; JANSSEN, I.; KAHN, H.S.; KATZMARZYK, P.T.; KUK, J.L.; SEIDELL, J.C.; SNIJDER, M.B.; SORENSEN, T.I.; DESPRES, J.P. Does the relationship between waist circumference, morbidity and mortality depend on measurement protocol for waist circumference? Obesity Research, Baton Rouge, v.9, n.4, p.312-25, 2008.

ROSS, W.; MARFELL-JONES, M. Kinanthropometry. In: MacDOUGALL, J.; WENGER, H.; GREEN, H. (Eds.). Physiological of the elite athlete. New York: Mouvement, 1983. p.75-115.

ROYSTON, P.; WRIGHT, E.M. Goodness-of-fit statistics for age-specific reference intervals. Statistics in Medicine, Chichester, v.19, p.2943-62, 2000.

SILVA, S.; MAIA, J.; CLAESSENS, A.L.; BEUNEN, G.; PAN, H. Growth references for Brazilian children and adolescents: healthy growth in Cariri study. Annals of Human Biology, London, v.39, p.11-18, 2011.

SOUSA, M.A.C.; MAIA, J.A.R. Crescimento somático, actividade física e aptidão física associada à saúde: um estudo populacional nas crianças do $1^{\circ}$ ciclo do ensino básico do Concelho de Amarante. Amarante: Câmara Municipal de Amarante/Faculdade de Desporto da Universidade do Porto, 2005.

ULIJASZEK, S. The international growth standard for children and adolescents project: environmental influences on preadolescent and adolescents growth in weight and height. Food and Nutrition Bulletin, Tokyo, v.27, n.4, p.S279-94, 2006. VAN BUUREN, S.; FREDRIKS, M. Worm plot: a simple diagnostic device for modelling growth reference curves. Statistics in Medicine, Chichester, v.20, p.1259-77, 2001.

ZIMMET, P.; ALBERTI, G.; KAUFMAN, F.; TAJIMA, N.; SILINK, M.; ARSLANIAN, S.; WONG, G.; BENNETT, P.; SHAW, J.; CAPRIO, S. The metabolic syndrome in children and adolescents. Lancet, New York, v.369, p.2059-61, 2007.

ANEXO I - Valores de L, M e S ( \pm erros-padrão) dos modelos mais parcimoniosos das curvas percentílicas da altura, peso, IMC e PC (masculino e feminino).

\begin{tabular}{|c|c|c|c|c|c|c|}
\hline \multirow{3}{*}{ Idade } & \multicolumn{3}{|c|}{ Masculino } & \multicolumn{3}{|c|}{ Feminino } \\
\hline & $\mathbf{L} \pm$ ep & $\mathbf{M} \pm \mathrm{ep}$ & $\boldsymbol{S}_{ \pm \mathrm{ep}}$ & $\mathbf{L} \pm$ ep & $\mathbf{M} \pm$ ep & $\boldsymbol{S}_{ \pm \mathrm{ep}}$ \\
\hline & \multicolumn{6}{|c|}{ Altura } \\
\hline 6 & $-0,04 \pm 1,51$ & $118,51 \pm 0,63$ & $0,04 \pm 0,003$ & $-0,20 \pm 1,69$ & $117,00 \pm 0,64$ & $0,04 \pm 0,002$ \\
\hline 7 & $-0,18 \pm 0,81$ & $123,13 \pm 0,28$ & $0,04 \pm 0,001$ & $-0,19 \pm 0,87$ & $122,01 \pm 0,27$ & $0,04 \pm 0,001$ \\
\hline 8 & $-0,39 \pm 0,61$ & $128,90 \pm 0,26$ & $0,04 \pm 0,001$ & $-0,44 \pm 0,63$ & $128,03 \pm 0,27$ & $0,04 \pm 0,001$ \\
\hline 9 & $-0,45 \pm 0,52$ & $134,55 \pm 0,23$ & $0,04 \pm 0,001$ & $-1,21 \pm 0,54$ & $133,69 \pm 0,25$ & $0,04 \pm 0,001$ \\
\hline \multirow[t]{2}{*}{10} & $-0,51 \pm 0,71$ & $139,66 \pm 0,29$ & $0,04 \pm 0,001$ & $-2,09 \pm 0,71$ & $140,32 \pm 0,34$ & $0,05 \pm 0,002$ \\
\hline & \multicolumn{6}{|c|}{ Peso } \\
\hline 6 & $-0,59 \pm 0,40$ & $22,92 \pm 0,48$ & $0,13 \pm 0,009$ & $-0,50 \pm 0,44$ & $22,00 \pm 0,53$ & $0,13 \pm 0,010$ \\
\hline 7 & $-0,79 \pm 0,22$ & $25,46 \pm 0,23$ & $0,16 \pm 0,005$ & $-0,74 \pm 0,22$ & $24,80 \pm 0,23$ & $0,16 \pm 0,005$ \\
\hline 8 & $-0,93 \pm 0,15$ & $28,97 \pm 0,24$ & $0,18 \pm 0,004$ & $-0,89 \pm 0,15$ & $28,62 \pm 0,25$ & $0,18 \pm 0,004$ \\
\hline 9 & $-0,88 \pm 0,12$ & $32,51 \pm 0,24$ & $0,19 \pm 0,004$ & $-0,84 \pm 0,13$ & $32,09 \pm 0,25$ & $0,19 \pm 0,004$ \\
\hline \multirow[t]{2}{*}{10} & $-0,74 \pm 0,16$ & $36,06 \pm 0,33$ & $0,20 \pm 0,006$ & $-0,79 \pm 0,19$ & $36,67 \pm 0,35$ & $0,19 \pm 0,006$ \\
\hline & \multicolumn{6}{|c|}{ IMC } \\
\hline 6 & $-1,76 \pm 0,43$ & $16,00 \pm 0,20$ & $0,07 \pm 0,006$ & $-1,06 \pm 0,60$ & $16,12 \pm 0,21$ & $0,10 \pm 0,005$ \\
\hline 7 & $-1,78 \pm 0,32$ & $16,56 \pm 0,09$ & $0,10 \pm 0,003$ & $-1,35 \pm 0,32$ & $16,74 \pm 0,10$ & $0,11 \pm 0,003$ \\
\hline 8 & $-1,80 \pm 0,19$ & $17,32 \pm 0,10$ & $0,12 \pm 0,003$ & $-1,55 \pm 0,22$ & $17,39 \pm 0,09$ & $0,13 \pm 0,002$ \\
\hline 9 & $-1,81 \pm 0,14$ & $17,90 \pm 0,09$ & $0,13 \pm 0,003$ & $-1,51 \pm 0,18$ & $18,01 \pm 0,09$ & $0,14 \pm 0,003$ \\
\hline \multirow[t]{2}{*}{10} & $-1,81 \pm 0,22$ & $18,62 \pm 0,12$ & $0,14 \pm 0,004$ & $-1,40 \pm 0,23$ & $18,67 \pm 0,13$ & $0,15 \pm 0,004$ \\
\hline & \multicolumn{6}{|c|}{ PC } \\
\hline 6 & $-2,98 \pm 0,90$ & $54,34 \pm 0,50$ & $0,06 \pm 0,004$ & $-2,15 \pm 0,62$ & $53,88 \pm 0,58$ & $0,06 \pm 0,005$ \\
\hline 7 & $-3,21 \pm 0,47$ & $56,62 \pm 0,24$ & $0,07 \pm 0,002$ & $-1,84 \pm 0,45$ & $55,69 \pm 0,25$ & $0,07 \pm 0,002$ \\
\hline 8 & $-3,06 \pm 0,32$ & $59,98 \pm 0,29$ & $0,09 \pm 0,003$ & $-1,48 \pm 0,28$ & $58,59 \pm 0,30$ & $0,09 \pm 0,003$ \\
\hline 9 & $-2,33 \pm 0,28$ & $63,56 \pm 0,32$ & $0,11 \pm 0,003$ & $-1,13 \pm 0,24$ & $62,03 \pm 0,33$ & $0,10 \pm 0,003$ \\
\hline 10 & $-1,85 \pm 0,35$ & $65,91 \pm 0,42$ & $0,12 \pm 0,004$ & $-0,79 \pm 0,37$ & $64,57 \pm 0,45$ & $0,11 \pm 0,004$ \\
\hline
\end{tabular}

624 • Rev. bras. Educ. Fís. Esporte, São Paulo, v.26, n.4, p.611-25, out./dez. 2012 
Cartas de referência do crescimento somático de crianças

ENDERECO

Mafalda Sofia Roriz

Laboratório de Cineantropometria e Estatística Aplicada

Faculdade de Desporto

Universidade do Porto

Recebido para publicação: 20/04/2012

R. Dr. Plácido Costa, 91

Aceito: 20/06/2012

4200-450 - Porto - PORTUGAL

e-mail:msroriz@hotmail.com

Rev. bras. Educ. Fís. Esporte, São Paulo, v.26, n.4, p.611-25, out./dez. 2012 • 625 